\title{
ON TENSOR PRODUCTS OF POLYNOMIAL REPRESENTATIONS
}

\author{
KEVIN PURBHOO AND STEPHANIE VAN WILLIGENBURG
}

\begin{abstract}
We determine the necessary and sufficient combinatorial conditions for which the tensor product of two irreducible polynomial representations of $G L(n, \mathbb{C})$ is isomorphic to another. As a consequence we discover families of LittlewoodRichardson coefficients that are non-zero, and a condition on Schur non-negativity.
\end{abstract}

\section{INTRODUCTION}

It is well known that the representation theory of $G L(n, \mathbb{C})$ is intimately connected to the combinatorics of partitions [7, Chapter 7: Appendix 2]. Before we address the main problem in this paper that concerns the representations of $G L(n, \mathbb{C})$, we will briefly review this connection.

Recall a partition $\lambda$ of a positive integer $m$, denoted $\lambda \vdash m$, is a list of positive integers $\lambda_{1} \geq \lambda_{2} \geq \cdots \geq \lambda_{\ell(\lambda)}>0$ whose sum is $m$. We call $m$ the size of $\lambda$, the $\lambda_{i}$ the parts of $\lambda$ and $\ell(\lambda)$ the length of $\lambda$. We also let $\lambda=0$ be the unique partition of 0 , called the empty partition of length 0 . Every partition corresponds naturally to a (Ferrers) diagram of shape $\lambda$, which consists of an array of $m$ boxes such that there are $\lambda_{i}$ left justified boxes in row $i$, where the rows are read from top to bottom. By abuse of notation we also denote this diagram by $\lambda$. In the following example the boxes are denoted by $\times$.

Example 1.1.

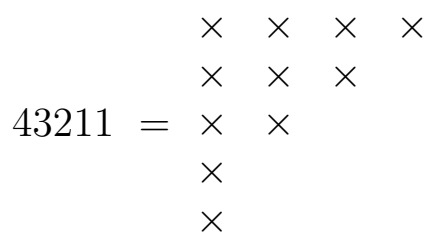

Moreover, given partitions $\lambda, \mu$ such that $\lambda_{i} \geq \mu_{i}$ for all $1 \leq i \leq \ell(\mu)$, if we consider the boxes of $\mu$ to be situated in the top left corner of $\lambda$ then we say that $\mu$ is a subdiagram of $\lambda$, and the skew diagram of shape $\lambda / \mu$ is the array of boxes contained in $\lambda$ but not in $\mu$. Again we abuse notation and denote this skew diagram by $\lambda / \mu$.

2000 Mathematics Subject Classification. 05E05, 05E10, 20C30.

Key words and phrases. polynomial representation, symmetric function, Littlewood-Richardson coefficient, Schur non-negative.

The authors were supported in part by the National Sciences and Engineering Research Council of Canada. 
Example 1.2.

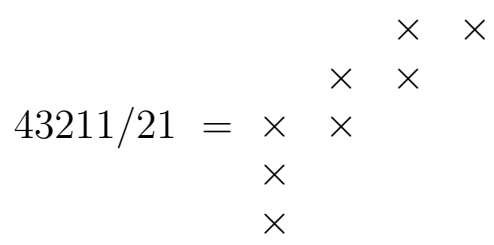

Furthermore, given a (skew) diagram, we can fill the boxes with positive integers to form a tableau $T$ and if $T$ contains $c_{1}(T) 1 \mathrm{~s}, c_{2}(T) 2 \mathrm{~s}, \ldots$ then we say it has content $c(T)=c_{1}(T) c_{2}(T) \cdots$. With this in mind we are able to state the connection between $G L(n, \mathbb{C})$ and partitions of $n$ as follows.

The irreducible polynomial representations $\phi^{\lambda}$ of $G L(n, \mathbb{C})$ are indexed by partitions $\lambda$ such that $\ell(\lambda) \leq n$ and given two irreducible polynomial representations of $G L(n, \mathbb{C}), \phi^{\mu}$ and $\phi^{\nu}$, one has

$$
\operatorname{char}\left(\phi^{\mu} \otimes \phi^{\nu}\right)=\sum_{\substack{\lambda \\ \ell(\lambda) \leq n}} c_{\mu \nu}^{\lambda} \operatorname{char} \phi^{\lambda}
$$

where $c_{\mu \nu}^{\lambda}$ is the number of tableaux $T$ of shape $\lambda / \mu$ such that

(i) the entries in the rows weakly increase from left to right

(ii) the entries in the columns strictly increase from top to bottom

(iii) $c(T)=\nu_{1} \nu_{2} \cdots$

(iv) when we read the entries from right to left and top to bottom the number of $i \mathrm{~s}$ we have read is always greater than or equal to the number of $(i+1) \mathrm{s}$ we have read.

This method for computing the $c_{\mu \nu}^{\lambda}$ is called the Littlewood-Richardson rule. As one might expect the $c_{\mu \nu}^{\lambda}$ are called Littlewood-Richardson coefficients. Observe we could have equally well chosen conditions (i)-(iv) to read

(i) the entries in the rows weakly increase from right to left

(ii) the entries in the columns strictly increase from bottom to top

(iii) $c(T)=\nu_{1} \nu_{2} \cdots$

(iv) when we read the entries from left to right and bottom to top the number of $i \mathrm{~s}$ we have read is always greater than or equal to the number of $(i+1) \mathrm{s}$ we have read.

For convenience we will call this the reverse Littlewood-Richardson rule.

Example 1.3. To illustrate both rules we now compute $c_{21,21}^{321}$. We will replace each box with the number it contains.

Using the Littlewood-Richardson rule we obtain $c_{21,21}^{321}=2$ from the tableaux ${ }_{1}{ }^{2}{ }^{1}$ and ${ }_{2} 1^{1}$. Meanwhile, using the reverse Littlewood-Richardson rule we also obtain $c_{21,21}^{321}=2$ from the tableaux ${ }_{1}{ }^{2}{ }^{1}$ and ${ }_{1}{ }^{2}$. 
Another place where Littlewood-Richardson coefficients arise is in the algebra of symmetric functions $\Lambda=\oplus_{m \geq 0} \Lambda^{m}$, which is a subalgebra of $\mathbb{Z}\left[\left[x_{1}, x_{2}, \ldots\right]\right]$ invariant under the natural action of the symmetric group. Each $\Lambda^{m}$ is spanned by $\left\{s_{\lambda}\right\}_{\lambda \vdash m}$ where $s_{0}:=1$ and

$$
s_{\lambda}:=\sum_{T} x^{T}
$$

The sum is over all tableaux $T$ that satisfy conditions (i) and (ii) of the LittlewoodRichardson rule and $x^{T}:=\prod_{i} x_{i}^{c_{i}(T)}$. For partitions $\lambda, \mu, \nu$ the structure coefficients of these Schur functions satisfy

$$
s_{\mu} s_{\nu}=\sum_{\lambda} c_{\mu \nu}^{\lambda} s_{\lambda}
$$

where the $c_{\mu \nu}^{\lambda}$ are again Littlewood-Richardson coefficients.

Similarly we can define the algebra of symmetric polynomials on $n$ variables by setting $x_{n+1}=x_{n+2}=\cdots=0$ above and working with Schur polynomials $s_{\lambda}\left(x_{1}, \ldots, x_{n}\right)$. Observe that by the definition (11) if $\ell(\lambda)>n$ then $s_{\lambda}\left(x_{1}, \ldots, x_{n}\right)=0$. The motivation for restricting to $n$ variables is that the irreducible representations of $G L(n, \mathbb{C})$ can be indexed such that

$$
\operatorname{char} \phi^{\lambda}=s_{\lambda}\left(x_{1}, \ldots, x_{n}\right) .
$$

See [2, 7] for further details.

\section{IDENTICAL TENSOR PRODUCTS}

We now begin to address the main problem of the paper, that is, to determine for which partitions $\lambda, \mu, \nu, \rho$ we have

$$
\phi^{\lambda} \otimes \phi^{\mu} \cong \phi^{\nu} \otimes \phi^{\rho}
$$

for irreducible polynomial representations of $G L(n, \mathbb{C})$.

For ease of notation, we assume $n$ is fixed throughout the remainder of the paper. Additionally, since $s_{\lambda}\left(x_{1}, \ldots, x_{n}\right)=0$ for $\ell(\lambda)>n$, we assume that all partitions have at most $n$ parts. We extend our partitions to exactly $n$ parts by appending a string of $n-\ell(\lambda) 0 \mathrm{~s}$. For example, if $n=4$ then $\lambda=32$ becomes $\lambda=3200$.

We now define an operation on diagrams that will be useful later.

Definition 2.1. Given partitions $\lambda$ and $\mu$ and an integer $s$ such that $0 \leq s \leq n-1$, the $s$-cut of $\lambda$ and $\mu$ is the partition whose parts are

$$
\begin{gathered}
\lambda_{1}+\mu_{1}, \lambda_{2}+\mu_{2}, \ldots, \lambda_{s}+\mu_{s}, \\
\lambda_{s+1}+\mu_{n}, \lambda_{s+2}+\mu_{n-1}, \ldots, \lambda_{n-1}+\mu_{s+2}, \lambda_{n}+\mu_{s+1}
\end{gathered}
$$

listed in weakly decreasing order.

Remark 2.1. Diagrammatically we can think of the $s$-cut of $\lambda$ and $\mu$ as 
(i) aligning the top rows of $\lambda$ and $\mu$ then

(ii) cutting the diagrams $\lambda$ and $\mu$ between the $s$ and $s+1$ rows

(iii) taking the rows of $\mu$ ( or $\lambda$ ) below the cut and rotating them by $180^{\circ}$

(iv) appending the newly aligned rows and sorting into weakly decreasing row length to make a diagram.

Example 2.2. If $n=6$, then the 2-cut of 432110 and 543200 is 973321 . This example can be viewed diagrammatically as the following.

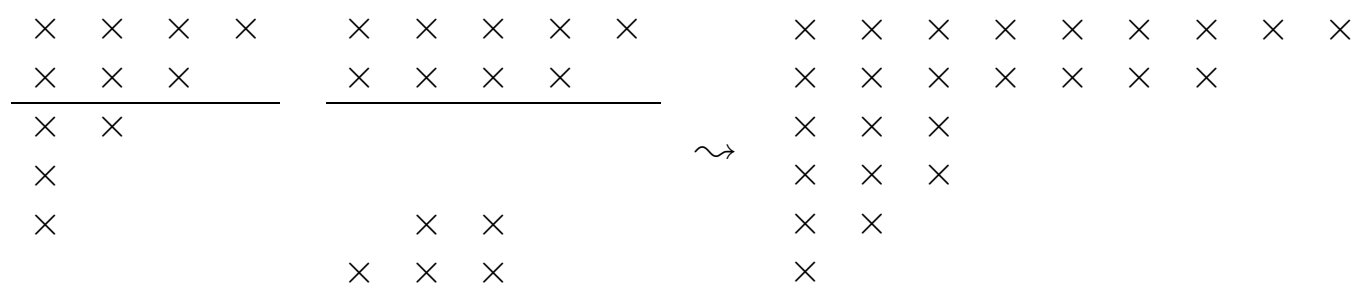

It transpires that the $s$-cut of $\lambda$ and $\mu$ yields a condition on Littlewood-Richardson coefficients.

Lemma 2.2. If $\lambda, \mu$ and $s$ are as in Definition 2.1 and $\kappa$ is the s-cut of $\lambda$ and $\mu$ then $c_{\lambda \mu}^{\kappa}>0$.

Proof. Observe that since the Littlewood-Richardson and reverse Littlewood Richardson rule yield the same coefficients there must be a bijection, $\psi$, between the tableaux generated by each. This bijection will play a key role in the proof.

Consider creating a tableau $T$ of shape $\kappa / \lambda$ where $\kappa_{i}=\lambda_{i}+\mu_{i}$ for $1 \leq i \leq s$ that will contribute towards the coefficient $c_{\lambda \mu}^{\kappa}$. If we use the Littlewood-Richardson rule then it is clear that for $1 \leq i \leq s$ we must fill the boxes of the $i$-th row with the $\mu_{i} i \mathrm{~s}$. Now all that remains for us to do is to fill the remaining boxes of $T$ with $\mu_{s+1}(s+1) \mathrm{s}, \ldots, \mu_{n} n \mathrm{~s}$. To do this we create a tableau $T^{\prime}$ of shape $\kappa_{s+1} \cdots \kappa_{n} / \lambda_{s+1} \cdots \lambda_{n}=\kappa / \kappa_{1} \cdots \kappa_{s} \lambda_{s+1} \cdots \lambda_{n}$ that will contribute towards the coefficient $c_{\alpha \beta}^{\gamma}$ where $\alpha=\lambda_{s+1} \cdots \lambda_{n}, \beta=\mu_{s+1} \cdots \mu_{n}$ and $\gamma=\kappa_{s+1} \cdots \kappa_{n}$. We do this as follows.

Fill the box at the bottom of each column from left to right with $\mu_{s+1} 1 \mathrm{~s}$. Then repeat on the remaining boxes with the $\mu_{s+2} 2 \mathrm{~s}$. Iterate this procedure until the boxes are full. Observe by the reverse Littlewood-Richardson rule that this filling contributes 1 to the coefficient $c_{\alpha \beta}^{\gamma}$. Now using $\psi$, create a tableau $T^{\prime \prime}$ of the same shape that satisfies the Littlewood-Richardson rule and increase each entry by $s$, forming a tableau $T^{\prime \prime \prime}$. Placing the entries of $T^{\prime \prime \prime}$ in the naturally corresponding boxes of $T$ we see we have a tableau that contributes 1 to the coefficient $c_{\lambda \mu}^{\kappa}$ by the Littlewood-Richardson rule and indeed $c_{\lambda \mu}^{\kappa}>0$.

Definition 2.3. If $\lambda, \mu$ and $s$ are as in Definition 2.1 then the s-poset of $\lambda$ and $\mu$ is the set of all partitions $\kappa$ such that

(i) $c_{\lambda \mu}^{\kappa}>0$ 
(ii) $\kappa_{i}=\lambda_{i}+\mu_{i}$ for all $1 \leq i \leq s$,

which are ordered lexicographically, that is, $\kappa>\kappa^{\prime}$ if and only if there exists some $i$, where $1 \leq i \leq n$, such that $\kappa_{1}=\kappa_{1}^{\prime}, \ldots, \kappa_{i-1}=\kappa_{i-1}^{\prime}$ and $\kappa_{i}>\kappa_{i}^{\prime}$.

Lemma 2.4. If $\lambda, \mu$ and $s$ are as in Definition 2.1 then the $s$-cut of $\lambda$ and $\mu$ is the unique minimal element in the s-poset of $\lambda$ and $\mu$.

Proof. Let $\xi$ be any element in the $s$-poset of $\lambda$ and $\mu$ and let $U$ be any tableau that will contribute towards the coefficient $c_{\lambda \mu}^{\xi}$ via the Littlewood-Richardson rule. As in the proof of Lemma 2.2 it is clear that for $1 \leq j \leq s$ we have that $j$ appears in every box of row $j$. Now consider the natural subtableau of shape $\xi_{s+1} \cdots \xi_{n} / \lambda_{s+1} \cdots \lambda_{n}$, which we denote by $\bar{U}$. Note that if we subtract $s$ from every entry in $\bar{U}$ then we obtain a tableau that contributes towards $c_{\left(\lambda_{s+1} \cdots \lambda_{n}\right)\left(\mu_{s+1} \cdots \mu_{n}\right)}^{\left(\xi_{s+1} \cdots \xi_{n}\right)}$ via the LittlewoodRichardson rule. If we then apply the bijection $\psi$ to rearrange these new entries, we obtain a tableau $U^{\prime}$ that contributes towards $c_{\left(\lambda_{s+1} \cdots \lambda_{n}\right)\left(\mu_{s+1} \cdots \mu_{n}\right)}^{\left(\xi_{s+1} \cdots \xi_{n}\right)}$ via the reverse Littlewood-Richardson rule.

Now let $\kappa$ be the $s$-cut of $\lambda$ and $\mu$. Let $T$ and $T^{\prime}$ be the tableaux constructed in the proof of Lemma 2.2. Recall that $T$ contributes towards the coefficient $c_{\lambda \mu}^{\kappa}$ via the Littlewood-Richardson rule, and that $T^{\prime}$ contributes towards $c_{\left(\lambda_{s+1} \cdots \lambda_{n}\right)\left(\mu_{s+1} \cdots \mu_{n}\right)}^{\left(\kappa_{s+1} \cdots \kappa_{n}\right)}$ via the reverse Littlewood-Richardson rule.

We now consider transforming $T^{\prime}$ into $U^{\prime}$ as follows. Since $T^{\prime}$ and $U^{\prime}$ both have content $\mu$, we can map the boxes of $T^{\prime}$ bijectively to the boxes of $U^{\prime}$ such that the $k$-th box containing $i$ from the left in $T^{\prime}$ maps to the $k$-th box containing $i$ from the left in $U^{\prime}$. This bijection factors as follows. First move each box in $T^{\prime}$ horizontally, so that it is in the same column as the corresponding box in $U^{\prime}$. Then move each box vertically to form $U^{\prime}$. By the construction of $T^{\prime}$ the entries are as left justified and low as possible, and so this transformation necessarily moves each box rightwards and upwards. It follows that $\kappa$, the shape of $T^{\prime}$, is lexicographically less than or equal to $\xi$, the shape of $U^{\prime}$, and we are done.

Recall that $\lambda_{n}$ is the number of columns of length $n$ in the diagram $\lambda$, and thus $\left(\lambda_{n}\right)^{n}$ is a subdiagram of $\lambda$. Define $\lambda^{-}:=\lambda /\left(\lambda_{n}\right)^{n}$. Notice that $\lambda^{-}$is a Ferrers diagram, with at most $n-1$ rows, and the number of columns of length $n-1$ is $\lambda_{n-1}^{-}$. We therefore define $\lambda^{--}:=\lambda^{-} /\left(\lambda_{n-1}^{-}\right)^{n-1}$. Notice that by (11) we have the factorization

$$
s_{\lambda}\left(x_{1}, \ldots, x_{n}\right)=\left(x_{1} \cdots x_{n}\right)^{\lambda_{n}} s_{\lambda^{-}}\left(x_{1}, \ldots, x_{n}\right),
$$

and moreover $x_{1} \cdots x_{n}$ does not divide $s_{\lambda^{-}}\left(x_{1}, \ldots, x_{n}\right)$.

Theorem 2.5. $\phi^{\lambda} \otimes \phi^{\mu} \cong \phi^{\nu} \otimes \phi^{\rho}$ as representations of $G L(n)$ if and only if $\lambda_{n}+\mu_{n}=$ $\nu_{n}+\rho_{n}$ and $\left\{\lambda^{-}, \mu^{-}\right\}=\left\{\nu^{-}, \rho^{-}\right\}$as multisets.

Proof. We will show that

$$
s_{\lambda}\left(x_{1}, \ldots, x_{n}\right) s_{\mu}\left(x_{1}, \ldots, x_{n}\right)=s_{\nu}\left(x_{1}, \ldots, x_{n}\right) s_{\rho}\left(x_{1}, \ldots, x_{n}\right)
$$


if and only if $\lambda_{n}+\mu_{n}=\nu_{n}+\rho_{n}$ and $\left\{\lambda^{-}, \mu^{-}\right\}=\left\{\nu^{-}, \rho^{-}\right\}$. The theorem then follows, using (2).

One direction is immediate. Suppose $\lambda_{n}+\mu_{n}=\nu_{n}+\rho_{n}$ and $\left\{\lambda^{-}, \mu^{-}\right\}=\left\{\nu^{-}, \rho^{-}\right\}$, then by (4) we have

$$
\begin{aligned}
s_{\lambda}\left(x_{1}, \ldots, x_{n}\right) s_{\mu}( & \left.x_{1}, \ldots, x_{n}\right) \\
= & \left(x_{1} \cdots x_{n}\right)^{\lambda_{n}+\mu_{n}} s_{\lambda^{-}}\left(x_{1}, \ldots, x_{n}\right) s_{\mu^{-}}\left(x_{1}, \ldots, x_{n}\right) \\
=\left(x_{1} \cdots x_{n}\right)^{\nu_{n}+\rho_{n}} s_{\nu^{-}}\left(x_{1}, \ldots, x_{n}\right) & s_{\rho^{-}}\left(x_{1}, \ldots, x_{n}\right) \\
& =s_{\nu}\left(x_{1}, \ldots, x_{n}\right) s_{\rho}\left(x_{1}, \ldots, x_{n}\right) .
\end{aligned}
$$

For the opposite direction, assume that (5) holds. We first show that $\lambda_{n}+\mu_{n}=$ $\nu_{n}+\rho_{n}$. If they were not equal, say $\lambda_{n}+\mu_{n}>\nu_{n}+\rho_{n}$, then by (41), we would have $\left(x_{1} \cdots x_{n}\right)^{\lambda_{n}+\mu_{n}-\nu_{n}-\rho_{n}} s_{\lambda^{-}}\left(x_{1}, \ldots, x_{n}\right) s_{\mu^{-}}\left(x_{1}, \ldots, x_{n}\right)=s_{\nu^{-}}\left(x_{1}, \ldots, x_{n}\right) s_{\rho^{-}}\left(x_{1}, \ldots, x_{n}\right)$, which is impossible since $x_{1} \cdots x_{n}$ does not divide the right hand side. Similarly we cannot have $\lambda_{n}+\mu_{n}<\nu_{n}+\rho_{n}$. Thus, we see furthermore that

$$
s_{\lambda^{-}}\left(x_{1}, \ldots, x_{n}\right) s_{\mu^{-}}\left(x_{1}, \ldots, x_{n}\right)=s_{\nu^{-}}\left(x_{1}, \ldots, x_{n}\right) s_{\rho^{-}}\left(x_{1}, \ldots, x_{n}\right) .
$$

Let $S(n)$ be the assertion that the equation (6) holds only if $\left\{\lambda^{-}, \mu^{-}\right\}=\left\{\nu^{-}, \rho^{-}\right\}$. To complete the proof of the theorem, it remains to show that $S(n)$ is true for all $n$. We prove this by induction.

The base case $n=1$ is trivial, since each of $\lambda^{-}, \mu^{-}, \nu^{-}, \rho^{-}$is necessarily the empty partition.

Now assume that $S(1), \ldots, S(n-1)$ are true. In particular this assumption implies that the theorem holds for smaller values of $n$. Furthermore, assume that (6) holds. Let

$$
\begin{array}{ll}
a:=\lambda_{n-1}^{-} & c:=\nu_{n-1}^{-} \\
b:=\mu_{n-1}^{-} & d:=\rho_{n-1}^{-} .
\end{array}
$$

Since (6) implies

$$
s_{\lambda^{-}}\left(x_{1}, \ldots, x_{n-1}\right) s_{\mu^{-}}\left(x_{1}, \ldots, x_{n-1}\right)=s_{\nu^{-}}\left(x_{1}, \ldots, x_{n-1}\right) s_{\rho^{-}}\left(x_{1}, \ldots, x_{n-1}\right),
$$

by our inductive hypothesis we must have

$$
a+b=c+d \quad \text { and } \quad\left\{\lambda^{--}, \mu^{--}\right\}=\left\{\nu^{--}, \rho^{--}\right\} .
$$

Assume without loss of generality that $\lambda^{--}=\nu^{--}=: \alpha$ and $\mu^{--}=\rho^{--}=: \beta$. To show that $\left\{\lambda^{-}, \mu^{-}\right\}=\left\{\nu^{-}, \rho^{-}\right\}$, we need to check that $a=c$ and $b=d$, or that $a=d$, $b=c$ and $\alpha=\beta$.

To show this we note that if (6) holds then for all $s, 0 \leq s \leq n-1$, the $s$-poset of $\lambda^{-}$and $\mu^{-}$, must be the same as the $s$-poset of $\nu^{-}$and $\rho^{-}$. Thus by Lemma 2.4, the $s$-cut of $\lambda^{-}$and $\mu^{-}$must be the same as the $s$-cut of $\nu^{-}$and $\rho^{-}$. 
The $s$-cut of $\lambda^{-}$and $\mu^{-}$has part sizes

$$
\begin{array}{cl}
a+b+\alpha_{j}+\beta_{j} & 1 \leq j \leq s \\
a+b+\alpha_{s+j}+\beta_{n-j+1} & 2 \leq j \leq n-s-1 \\
a+\alpha_{s+1} & \\
b+\beta_{s+1} &
\end{array}
$$

whereas the $s$-cut of $\nu^{-}$and $\rho^{-}$has part sizes

$$
\begin{array}{cl}
a+b+\alpha_{j}+\beta_{j} & 1 \leq j \leq s \\
a+b+\alpha_{s+j}+\beta_{n-j+1} & 2 \leq j \leq n-s-1 \\
c+\alpha_{s+1} & \\
d+\beta_{s+1} . &
\end{array}
$$

These lists must agree. Consequently we must have

$$
a+\alpha_{s+1}=c+\alpha_{s+1} \quad \text { or } \quad a+\alpha_{s+1}=d+\beta_{s+1},
$$

for all $s$. If, for any $s$, we are in the first situation, then $a=c$ and $b=d$ as desired. If not then

$$
a+\alpha_{s+1}=d+\beta_{s+1} \quad \text { and } \quad c+\alpha_{s+1}=b+\beta_{s+1}
$$

for all $0 \leq s \leq n-1$. In particular, since $\alpha_{n-1}=\beta_{n-1}=0$, we have $a=d$ and $b=c$, ensuring $\alpha_{j}=\beta_{j}$ for $1 \leq j \leq n-1$.

Example 2.3. If $n=3$,

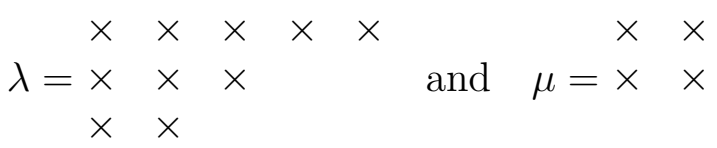

then $\phi^{\lambda} \otimes \phi^{\mu} \cong \phi^{\nu} \otimes \phi^{\rho}$ if and only if $\{\nu, \rho\}$ is equal to one of

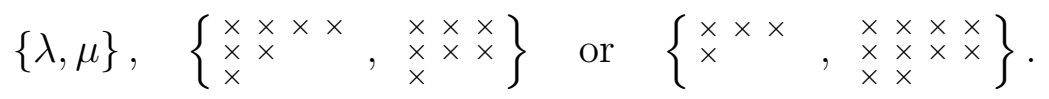

We consequently obtain a strict lower bound on $n$, in terms of the size of the partitions, to guarantee that (3) has only trivial solutions.

Corollary 2.6. Suppose $m, m^{\prime}$ are non-negative integers. If $n>\max \left\{m, m^{\prime}\right\}$, then for any partitions $\lambda \vdash m$ and $\mu \vdash m^{\prime}$, we have that

$$
\phi^{\lambda} \otimes \phi^{\mu} \cong \phi^{\nu} \otimes \phi^{\rho}
$$

has only the trivial solution $\{\nu, \rho\}=\{\lambda, \mu\}$. If $\min \left\{m, m^{\prime}\right\} \geq 2$ and $n \leq \max \left\{m, m^{\prime}\right\}$, then there exist $\lambda \vdash m$ and $\mu \vdash m^{\prime}$ for which (7) has non-trivial solutions. 


\section{SCHUR NON-NEGATIVITY}

A question that has received much attention recently, for example [4, 6], is the question of Schur non-negativity. The notion of Schur non-negativity is of interest as it arises in the study of algebraic geometry [1], quantum groups [3], and branching problems in representation theory [5].

One of the most basic Schur non-negativity questions is the following. Given partitions $\lambda, \mu, \nu, \rho$ when is the difference $s_{\lambda} s_{\mu}-s_{\nu} s_{\rho}$ a non-negative linear combination of Schur functions? Note that if $s_{\lambda} s_{\mu}-s_{\nu} s_{\rho}$ is Schur non-negative then the same is certainly true of the corresponding expression in finitely many variables

$$
s_{\lambda}\left(x_{1}, \ldots, x_{n}\right) s_{\mu}\left(x_{1}, \ldots, x_{n}\right)-s_{\nu}\left(x_{1}, \ldots, x_{n}\right) s_{\rho}\left(x_{1}, \ldots, x_{n}\right) .
$$

The following yields a test for failure of Schur non-negativity.

Corollary 3.1. For $0 \leq s \leq n-1$, let $\kappa=\kappa_{1} \ldots \kappa_{n}$ be the $s$-cut of $\lambda$ and $\mu$, and let $\xi=\xi_{1} \ldots \xi_{n}$ be the $s$-cut of $\nu$ and $\rho$. Form the sequences

$$
\sigma(s):=\kappa_{1} \ldots \kappa_{s} \xi_{s+1} \ldots \xi_{n} \text { and } \tau(s):=\xi_{1} \ldots \xi_{s} \kappa_{s+1} \ldots \kappa_{n} .
$$

If there exists an $s$ for which $\tau(s)$ is lexicographically greater than $\sigma(s)$, then

$$
s_{\lambda}\left(x_{1}, \ldots, x_{n}\right) s_{\mu}\left(x_{1}, \ldots, x_{n}\right)-s_{\nu}\left(x_{1}, \ldots, x_{n}\right) s_{\rho}\left(x_{1}, \ldots, x_{n}\right)
$$

is not Schur non-negative.

Proof. Suppose the $s$-cut of $\lambda$ and $\mu$ is not equal to the $s$-cut of $\nu$ and $\rho$, and let $k$ be the first index in which they differ. If $k \leq s$, and $\xi_{k}>\kappa_{k}$, then by the Littlewood-

Richardson rule, $c_{\lambda \mu}^{\xi}=0$. On the other hand if $k>s$ and $\kappa_{k}>\xi_{k}$, then the same is true by Lemma 2.4. In either case, by Lemma 2.2, $c_{\nu \rho}^{\xi}>0$, and thus

$$
s_{\lambda}\left(x_{1}, \ldots, x_{n}\right) s_{\mu}\left(x_{1}, \ldots, x_{n}\right)-s_{\nu}\left(x_{1}, \ldots, x_{n}\right) s_{\rho}\left(x_{1}, \ldots, x_{n}\right)
$$

is not Schur non-negative.

Example 3.1. Suppose $n=3$, and

$$
\begin{array}{ll}
\lambda=310 & \nu=220 \\
\mu=110 & \rho=200 .
\end{array}
$$

Then $\sigma(0)=222<321=\tau(0)$. Thus we can conclude that $s_{\lambda} s_{\mu}-s_{\nu} s_{\rho}$ is not Schur non-negative. On the other hand $\sigma(1)=420>411=\tau(1)$. Thus $s_{\nu} s_{\rho}-s_{\lambda} s_{\mu}$ is also not Schur non-negative.

\section{ACKnowledgements}

The authors are grateful to Matthew Morin for helpful discussions, Christopher Ryan for providing some data and Mark Skandera for suggesting the problem. They would also like to thank the referee for illuminating comments. 


\section{REFERENCES}

[1] S. Fomin, W. Fulton, C. Li and Y. Poon, Eigenvalues, singular values, and Littlewood-Richardson coefficients, Amer. J. Math., 127 (2005), 101-127.

[2] I. Macdonald, Symmetric Functions and Hall Polynomials, 2nd Edition, Oxford University Press, New York, USA, 1995.

[3] A. Lascoux, B. Leclerc and J.-Y. Thibon, Ribbon tableaux, Hall-Littlewood functions, quantum affine algebras and unipotent varieties, J. Math. Phys., 38 (1997), 1041-1068.

[4] T. Lam, A. Postnikov and P. Pylyavskyy, Schur positivity and Schur log-concavity, Amer. J. Math., to appear.

[5] A. Okounkov, Log-concavity of multiplicities with applications to characters of $U(\infty), \quad A d v$. Math., 127 no. 2 (1997), 258-282.

[6] B. Rhoades and M. Skandera, Kazhdan-Lusztig immanants and products of matrix minors, $J$. Alg, to appear.

[7] R. Stanley, Enumerative Combinatorics, Vol. 2, Cambridge Studies in Advanced Mathematics, Vol. 62, Cambridge University Press, Cambridge, UK, 1999.

Department of Mathematics, University of British Columbia, Vancouver, BC V6T $1 \mathrm{Z2}$

E-mail address: kevinp@math.ubc.ca

Department of Mathematics, University of British Columbia, Vancouver, BC V6T $1 \mathrm{Z2}$

E-mail address: steph@math.ubc.ca 